\title{
Actualidad del tratamiento farmacológico de la pulpitis irreversible sintomática
}

\author{
Current status of pharmacological treatment \\ of symptomatic irreversible pulpitis
}

Evelyn Mercedes Mamani Usecca ${ }^{1 a b}$

Darmely Helen Quispe Ticona ${ }^{1 \mathrm{ab}}$

Ulises Massino Peñaloza De La Torre ${ }^{1 \mathrm{~cd}}$
(1) https://orcid.org/0000-0002-4947-5926

https://orcid.org/0000-0002-1315-7117

iDttps://orcid.org/0000-0002-8510-2350

Correspondencia: upenalozat@unjbg.edu.pe

\section{Resumen}

En el presente artículo de revisión bibliográfica tenemos como objetivo dar a conocer recomendaciones para el tratamiento farmacológico sistémico y local de la pulpitis irreversible sintomática, la cual es una de las causas más frecuentes de visita al odontólogo por la búsqueda de un tratamiento para el dolor ocasionado. Según los artículos encontrados de bases de datos como PubMed, Cochrane, Science Direct, Google Scholar, además del consenso 2017 de la Sociedad Europea de Endodoncia (todos ellos con un alto nivel de evidencia científica), la premedicación con antiinflamatorios no esteroideos aumenta la tasa de éxito de la anestesia local proporcionando una anestesia predecible, la solución salina como irrigante a $2.5^{\circ} \mathrm{C}$ reduce el dolor posoperatorio, la prescripción de antibióticos está proscrita en biopulpectomías dado que no disminuye el dolor posoperatorio y los AINE son los fármacos de elección para el control del dolor postratamiento de conductos.

Palabras clave: manejo del dolor, pulpitis, endodoncia, tratamiento farmacológico (DeCS)

\begin{abstract}
In this literature review article, the objective is to present recommendations for the systemic and local pharmacological treatment of symptomatic irreversible pulpitis, which is one of the most frequent causes of visits to the dentist in search of a treatment for pain. According to articles found in databases such as PubMed, Cochrane, Science Direct, Google Scholar, in addition to the 2017 consensus of the European Society of Endodontics (all of them with a high level of scientific evidence), premedication with non-steroidal anti-inflammatory drugs increases the rate of success of local anesthesia providing predictable anesthesia, saline solution as an irrigant at $2.5^{\circ} \mathrm{C}$ reduces postoperative pain, the prescription of antibiotics is prohibited in biopulpectomies since it does not reduce postoperative pain and NSAIDs are the drugs of choice for the pain control after root canal treatment.
\end{abstract}

Keywords: pain management, pulpitis, endodontics, pharmacological treatment (MeSH NLM)

\footnotetext{
${ }^{1}$ Universidad Nacional Jorge Basadre Grohmann. Escuela Profesional de Odontología. Tacna, Perú

a Estudiante de pregrado de Odontología

${ }^{\mathrm{b}}$ Miembro del Semillero Odontología Basadrina

c Especialista en Periodoncia e Implantología

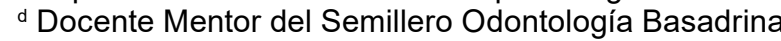




\section{Introducción}

En el tratamiento endodóntico de dientes vitales, la odontalgia es a menudo una situación de emergencia que requiere tratamiento inmediato. Incluso en esta pandemia de la COVID-19, las emergencias endodónticas son muy comunes $(97.90 \%$ ), de las cuales el $53.13 \%$ es por el dolor producido por la pulpitis irreversible sintomática. ${ }^{1}$ Este dolor asociado con la pulpitis suele ser intenso y difícil de controlar, incluso con analgésicos, es por eso que se llega a usar corticoides. ${ }^{2}$ El tipo de tratamiento endodóntico a realizar es la biopulpectomía para tratar la pulpitis. Existe evidencia que el dolor puede continuar después de realizar la endodoncia. EI 59-61\% de los pacientes experimentan dolor de moderado a severo en el día 1 del posoperatorio y el 50-57\% de los pacientes el día 2, y los índices de dolor van disminuyendo los 2 días siguientes. ${ }^{3}$

El dolor siempre ha sido un factor que ocupa gran importancia en este tipo de tratamientos; paralelamente existe una controversia ¿cómo debe ser la medicación sistémica y local para tratar la odontalgia originada por esta pulpitis?, ¿qué analgésicos son los más eficaces para tratar la odontalgia?, ¿lograremos la efectividad anestésica en el tratamiento endodóntico?, ¿recetamos antibióticos? Se ha encontrado que el $39.3 \%$ de los odontólogos informaron que recetarían antibióticos para la pulpitis irreversible ${ }^{4}$ entonces ¿los antibióticos reducirán el dolor de la pulpitis? Como medicación local ¿qué tipo de tratamiento químico auxiliar usaremos en las biopulpectomías?

En el presente artículo tenemos como objetivo hacer una revisión actualizada de la literatura sobre las indicaciones, uso de medicamentos locales y sistémicos para el tratamiento de pulpitis irreversible sintomática proponiendo recomendaciones basadas en evidencia científica, para lo cual se hizo una revisión bibliográfica en bases de datos como PubMed, Cochrane, Science Direct, Google Scholar, tomándose en cuenta revisiones sistemáticas, metaanálisis y ensayos clínicos. Además, se han tomado en cuenta artículos de la Sociedad Europea de Endodoncia.

\section{La pulpitis}

La etiología de la pulpitis se debe a factores como lesiones cariosas extensas, pérdida del material sellador de las restauraciones, así como también por traumatismos dentales o en casos en los que ocurra una fractura considerable de la pieza dentaria. ${ }^{5}$

\section{Características de su microbiota}

En una investigación donde se tomaron muestras de las piezas dentarias con lesiones cariosas profundas y con exposiciones pulpares y dientes diagnosticados con pulpitis se obtuvieron los tres phylus más comunes, componiendo el 98 \% de la microbiota; identificándose las Firmicutes, Actinobacteria y Proteobacteria, siendo los géneros más comunes Lactobacillus, Pseudoramibacter, Streptococcus, Stenotrophomonas, Veillonella, además de otros microrganismos dominados, en su mayoría, por el género Lactobacillus; ${ }^{6-8}$ además, este género ha sido relacionado con el dolor continuo; las especies de Streptococcus, Dialister invisus y P. micra, con el dolor punzante, y S. mutans como el microorganismo asociado con el dolor a la percusión. ${ }^{7}$ Además, estas especies son también encontradas en conductos radiculares infectados, en los cuales se requiere tratamientos más agresivos, excepto por Lactobacillus, el cual se encuentra mayormente en este estado pulpar. $^{6}$ 


\section{Anestésicos locales en pulpitis}

Lograr el efecto de los anestésicos locales en piezas vitales con inflamación, producto de una pulpitis irreversible, es un factor muy importante para el manejo del dolor. Está comprobado en un metaanálisis que el éxito de la anestesia pulpar mejora cuando se aumenta el volumen de la solución anestésica, cuando se prescribe de 30, 45 a 60 minutos antes de las biopulpectomías y se aplica en una dosis oral única la premedicación de un $\mathrm{AINE}^{9,10}$ como la indometacina, meloxicam, peroxicam y diclofenaco potásico, conjuntamente con paracetamol o un opioide. También se halló que el uso de articaína como anestésico local ayuda a lograr el éxito anestésico; esto se debe a su actividad química que permite que la solución se difunda al ápice de los dientes, ${ }^{10}$ la administración de ibuprofeno de $600 \mathrm{mg} 1$ hora antes como premedicación genera éxito en el bloqueo del nervio alveolar inferior, ya que reduce el riesgo del dolor después del efecto anestésico. ${ }^{11}$

\section{Auxiliares químicos de la biopulpectomía}

El hipoclorito de sodio no deja de ser la sustancia preferida para la irrigación de conductos; las concentraciones de hipoclorito de sodio de $0.5 \%, 1 \%, 2.5 \%$, son las más indicadas en tratamientos de dientes vitales, si se usa en otras concentraciones puede ser muy citotóxico, causar quemaduras de los tejidos periapicales, edema, sangrado profuso y hematomas. ${ }^{12}$ También se usa la solución salina como irrigante, esta minimiza la irritación y no produce daños en los tejidos, ya que es una solución biológicamente compatible..$^{13}$

La medicación intraconducto se utiliza en caso que se aplique el régimen de visitas doble o múltiple, los apósitos empleados en piezas con pulpas vitales son hidróxido de calcio, formocresol diluido y, en algunos casos, antibióticos (Pasta TAP), ${ }^{14,15}$ aunque también la medicación con AINE o corticosteroides que alivian el dolor durante las 24 horas. $^{2}$ La Sociedad Europea de Endodoncia aboga por el uso de hidróxido de calcio, esto para evitar la decoloración de la dentina que pueda causar el uso de la Pasta TAP. ${ }^{16}$ En el caso del régimen de una sola visita se ha encontrado el éxito de la crioterapia irrigando solución salina a $2.5^{\circ} \mathrm{C}$ durante 5 minutos, como último irrigante después de la limpieza del conducto, el cual redujo el dolor posoperatorio en un $85.88 \%$ y se presenta como una medida simple, rentable y no tóxica. ${ }^{17}$

\section{Medicación sistémica de la pulpitis}

\section{Antibióticos}

La prescripción de antibióticos para tratar el dolor en la pulpitis es una mala práctica que se continúa dando por una gran mayoría de odontólogos, sabiendo aun que en la fase inicial de la pulpitis irreversible no existen signos clínicos de infección bacteriana. ${ }^{18,4}$ Esta prescripción errada de antibióticos, mayormente, se da en casos con odontólogos con poca experiencia. ${ }^{4}$ Este uso infundado de antibióticos está impulsando el aumento global de infecciones resistentes a los fármacos. ${ }^{18}$ La fisiopatología del dolor en las pulpitis irreversible es producida por una pulpa vital inflamada, no por una pulpa no vital infectada; por lo tanto, un antibiótico no solo no va a causar una disminución del dolor, sino que no va a tratar una infección que no existe; sumado a que en las pulpas vitales hay un suministro de sangre normal, solo una pequeña cantidad de antibiótico llega a la pulpa. ${ }^{19}$ En la declaración de la Sociedad Europea de Endodoncia del año 2017 se señala que no está indicado el uso de antibióticos en la pulpitis irreversible ${ }^{20}$ y esta no debe considerarse como tratamiento farmacológico sustituto de la pulpectomía inmediata. ${ }^{18}$ 


\section{Analgésicos}

El manejo del dolor genera en el paciente un impacto positivo en el tratamiento endodóntico, es por eso que los odontólogos tienen que ser capaces de aliviar el dolor. ${ }^{2}$ Para tratar el dolor de una pulpitis se requiere el tratamiento inmediato de una pulpectomía, pero existen casos en los que este no se puede realizar de manera inmediata, por esto es que se han probado diferentes medidas para aliviar el dolor preoperatorio, como el uso de la acupuntura, ${ }^{21}$ así como también se viene probando la toma de corticosteroides para retrasar el tratamiento endodóntico. ${ }^{22}$

El dolor que se genera en la pulpitis, una vez realizado el desbridamiento de conductos, se lo relaciona con el número de visitas (una o múltiples) en las cuales se realiza la pulpectomía; respecto a esto, en la revisión sistemática realizada por Manfredi et al. y Cochrane Oral Health Group ${ }^{14}$ se concluyó que no hay pruebas que sugieran que el tratamiento de conductos (una sola visita o múltiples) sea mejor que el otro, ninguno puede prevenir las complicaciones a corto o largo plazo; ${ }^{14} \sin$ embargo, en ambos regímenes de visitas el dolor posoperatorio se presenta, en la mayoría de los casos, después del desbridamiento. Los AINE son los fármacos de elección para el control del dolor; ibuprofeno de 400-600 mg o este asociado al acetaminofén $600 \mathrm{mg} / 650 \mathrm{mg} ;{ }^{3,23}$ también se pueden usar corticosteroides preoperatorios por vía oral, los cuales son efectivos para reducir el dolor moderado o severo posoperatorio hasta 24 horas después de la pulpectomía, cuando el dolor se presenta más intenso. Usar $4 \mathrm{mg}$ de dexamentasona, ${ }^{2}$ así como también $40 \mathrm{mg}$ de prednisolona ${ }^{24,25}$ han mostrado efectividad y sin presencia de efectos adversos, al ser la administración de una sola dosis. ${ }^{25}$

\section{Conclusiones}

La premedicación con antiinflamatorios no esteroideos aumenta la tasa de éxito de la anestesia local proporcionando una anestesia predecible y un mayor control del dolor durante el tratamiento endodóntico de pulpitis irreversible. La solución salina como irrigante a $2.5^{\circ} \mathrm{C}$ reduce el dolor posoperatorio, la prescripción de antibióticos en biopulpectomías no disminuye el dolor posoperatorio, más bien impulsa el aumento global de resistencia bacteriana. Los AINE son los fármacos de elección para el control del dolor postratamiento de conductos.

\section{Recomendaciones}

Basados en la evidencia encontrada sobre medicación local y sistémica en los casos de pulpitis irreversible sintomática sugerimos la premedicación con AINE, 30 a 60 minutos antes de la administración del anestésico para la ejecución de la biopulpectomía; así como para la irrigación del conducto radicular, el uso de la crioterapia, aplicando solución salina a $2.5^{\circ} \mathrm{C}$ durante 5 minutos como último irrigante después de la limpieza del conducto, provocando una reducción del dolor posoperatorio en un $85.88 \%$; si no se puede realizar la biopulpectomía en una sesión, se recomienda como medicación intraconducto el uso de hidróxido de calcio, y para la medicación posoperatoria, el uso de antibióticos es indiscutiblemente innecesario, donde es suficiente la medicación con analgésicos como los AINE hasta 24 horas después de la biopulpectomía.

\section{Referencias}

1. Yu J, Zhang T, Zhao D, Haapasalo M, Shen Y. Characteristics of Endodontic Emergencies during Coronavirus Disease 2019 Outbreak in Wuhan. J Endod [Internet]. 2020 Jun 1 [cited 2021 Feb 15];46(6):730-5. Available from: /pmc/articles/PMC7151235/

2. Nogueira BML, Silva LG, Mesquita CRM, Menezes SAF, Menezes TOA, FariaAGM, et al. Is the Use of Dexamethasone Effective in Controlling Pain Associated with Symptomatic Irreversible Pulpitis? A Systematic Review [Internet]. Vol. 44, Journal of Endodontics. Elsevier Inc.; 2018 [cited 2021 Feb 10]. p. 703-10. Available from: https://pubmed.ncbi. nlm.nih.gov/29571913/ 
3. Stamos A, Drum M, Reader A, Nusstein J, Fowler S, Beck M. An Evaluation of Ibuprofen Versus Ibuprofen/ Acetaminophen for Postoperative Endodontic Pain in Patients With Symptomatic Irreversible Pulpitis and Symptomatic Apical Periodontitis. Anesth Prog [Internet]. 2019 Dec 1 [cited 2021 Feb 10];66(4):192-201. Available from: https:// pubmed.ncbi.nlm.nih.gov/31891295/

4. Agnihotry A, Gill KS, Stevenson RG, Fedorowicz Z, Kumar V, Sprakel J, et al. Irreversi bl e pulpitis - A source of antibiotic over-prescription? Braz Dent J [Internet]. 2019 Jul 1 [cited 2021 Feb 15];30(4):374-9. Available from: http:// dx.doi.org/10.1590/0103-6440201902873-com

5. Levin LG, Law AS, Holland GR, Abbott P V., Roda RS. Identify and Define All Diagnostic Terms for Pulpal Health and Disease States. J Endod [Internet]. 2009 [cited 2021 Feb 20];35(12):1645-57. Available from: https://pubmed.ncbi. nlm.nih.gov/19932339/

6. Rôças IN, Alves FRF, Rachid CTCC, Lima KC, Assunção I V., Gomes PN, et al. Microbiome of Deep Dentinal Caries Lesions in Teeth with Symptomatic Irreversible Pulpitis. PLoS One [Internet]. 2016 May 1 [cited 2021 Feb 20];11(5). Available from: /pmc/articles/PMC4852894/

7. Rôças IN, Lima KC, Assunção I V., Gomes PN, Bracks I V., Siqueira JF. Advanced Caries Microbiota in Teeth with Irreversible Pulpitis. J Endod [Internet]. 2015 Sep 1 [cited 2021 Feb 20];41(9):1450-5. Available from: https:// pubmed.ncbi.nlm.nih.gov/26187422/

8. Zheng J, Wu Z, Niu K, Xie Y, Hu X, Fu J, et al. Microbiome of Deep Dentinal Caries from Reversible Pulpitis to Irreversible Pulpitis. J Endod. 2019 Mar 1;45(3):302-309.e1.

9. Tupyota P, Chailertvanitkul P, Laopaiboon M, Ngamjarus C, Abbott P V., Krisanaprakornkit S. Supplementary techniques for pain control during root canal treatment of lower posterior teeth with irreversible pulpitis: A systematic review and meta-analysis. Aust Endod J [Internet]. 2018 Apr 1 [cited 2021 Feb 19];44(1):14-25. Available from: https://pubmed.ncbi.nlm.nih.gov/28736921/

10. Shirvani A, Shamszadeh S, Eghbal MJ, Marvasti LA, Asgary S. Effect of preoperative oral analgesics on pulpal anesthesia in patients with irreversible pulpitis-a systematic review and meta-analysis [Internet]. Vol. 21, Clinical Oral Investigations. Springer Verlag; 2017 [cited 2021 Feb 21]. p. 43-52. Available from: https://pubmed.ncbi.nlm. nih.gov/27837343/

11. De Geus JL, Wambier LM, Boing TF, Loguercio AD, Reis A. Effect of ibuprofen on the efficacy of inferior alveolar nerve block in patients with irreversible pulpitis: A meta-analysis [Internet]. Vol. 45, Australian Endodontic Journal. Wiley-Blackwell Publishing Asia; 2019 [cited 2021 Feb 21]. p. 246-58. Available from: https://pubmed.ncbi.nlm.nih. gov/30295006/

12. De Estomatología F, Beltrán R, Betsabe L, Mondragón P. Universidad Peruana Cayetano Heredia “Medicación Intraconducto: Cómo, Cuándo y Porqué" Investigación Bibliográfica del Proceso de Suficiencia Profesional para Obtener el Título de Cirujano Dentista. Lima; 2009.

13. De Estomatología F, Beltrán R, Vanessa S, Abad B. Universidad Peruana Cayetano Heredia "Soluciones Irrigantes en Endodoncia" Investigación Bibliográfica del Proceso de Suficiencia Profesional para Obtener el Título de Cirujano Dentista. Lima; 2009.

14. Manfredi M, Figini L, Gagliani M, Lodi G. Single versus multiple visits for endodontic treatment of permanent teeth [Internet]. Vol. 2016, Cochrane Database of Systematic Reviews. John Wiley and Sons Ltd; 2016 [cited 2021 Jan 25]. Available from: /pmc/articles/PMC6463951/?report=abstract

15. Tirupathi SP, Krishna N, Rajasekhar S, Nuvvula S. Clinical Efficacy of Single-visit Pulpectomy over Multiple-visit Pulpectomy in Primary Teeth: A Systematic Review. Int J Clin Pediatr Dent [Internet]. 2019 Oct [cited 2021 Feb 22];12(5):453-9. Available from: /pmc/articles/PMC7229364/

16. Segura-Egea JJ, Gould K, Şen BH, Jonasson P, Cotti E, Mazzoni A, et al. European Society of Endodontology position statement: the use of antibiotics in endodontics. Int Endod J. 2018;51(1):20-5.

17. Keskin C, Özdemir Ö, Uzun İ, Güler B. Effect of intracanal cryotherapy on pain after single-visit root canal treatment. Aust Endod J [Internet]. 2017 Aug 1 [cited 2021 Feb 22];43(2):85-90. Available from: https://pubmed.ncbi.nlm.nih. gov/27699913/

18. Agnihotry A, Thompson W, Fedorowicz Z, van Zuuren EJ, Sprakel J. Antibiotic use for irreversible pulpitis [Internet]. Vol. 2019, Cochrane Database of Systematic Reviews. John Wiley and Sons Ltd; 2019 [cited 2021 Feb 10]. Available from: https://www.cochranelibrary.com/cdsr/doi/10.1002/14651858.CD004969.pub5/full

19. Hoskin E, Veitz-Keenan A. Antibiotics are not useful to reduce pain associated with irreversible pulpitis [Internet]. Vol. 17, Evidence-Based Dentistry. Nature Publishing Group; 2016 [cited 2021 Feb 15]. p. 81-2. Available from: https:// pubmed.ncbi.nlm.nih.gov/27767119/

20. Segura-Egea JJ, Gould K, Şen BH, Jonasson P, Cotti E, Mazzoni A, et al. European Society of Endodontology position statement: the use of antibiotics in endodontics [Internet]. Vol. 51, International Endodontic Journal. Blackwell Publishing Ltd; 2018 [cited 2021 Feb 19]. p. 20-5. Available from: https://pubmed.ncbi.nlm.nih.gov/28436043/

21. Murugesan H, Venkatappan S, Renganathan SK, Narasimhan S, Sekar M. Comparison of Acupuncture with Ibuprofen for Pain Management in Patients with Symptomatic Irreversible Pulpitis: A Randomized Double-Blind Clinical Trial. JAMS J Acupunct Meridian Stud [Internet]. 2017 Dec 1 [cited 2021 Feb 21];10(6):396-401. Available from: https:// pubmed.ncbi.nlm.nih.gov/29275795/ 
22. Kérourédan O, Jallon L, Perez P, Germain C, Péli JF, Oriez D, et al. Efficacy of orally administered prednisolone versus partial endodontic treatment on pain reduction in emergency care of acute irreversible pulpitis of mandibular molars: Study protocol for a randomized controlled trial. Trials [Internet]. 2017 Mar 28 [cited 2021 Feb 15];18(1). Available from: /pmc/articles/PMC5371272/

23. Aminoshariae A, Kulild JC, Donaldson M, Hersh E V. Evidence-based recommendations for analgesic efficacy to treat pain of endodontic origin: A systematic review of randomized controlled trials [Internet]. Vol. 147, Journal of the American Dental Association. American Dental Association; 2016 [cited 2021 Feb 21]. p. 826-39. Available from: https://pubmed.ncbi.nlm.nih.gov/27475974/

24. Elkhadem A, Ezzat K, Ramadan M, AbdelGhaffar S, Khamis D, Hassan A, et al. The effect of preoperative oral administration of prednisolone on postoperative pain in patients with symptomatic irreversible pulpitis: a singlecentre randomized controlled trial. Int Endod J [Internet]. 2018 Apr 1 [cited 2021 Feb 21];51:e189-96. Available from: https://pubmed.ncbi.nlm.nih.gov/28560802/

25. Jalalzadeh SM, Mamavi A, Shahriari S, Santos FA, Pochapski MT. Effect of pretreatment prednisolone on postendodontic pain: A double-blind parallel-randomized clinical trial. J Endod [Internet]. 2010 [cited 2021 Feb 21];36(6):978-81. Available from: https://pubmed.ncbi.nlm.nih.gov/20478449/

- Conflicto de intereses: La presente investigación no presenta conflicto de intereses entre los investigadores.

- Fuente de financiamiento: La presente investigación fue financiada por los investigadores. 